\title{
Perspectiva de géneros y feminismos jurídicos en la enseñanza del derecho
}

\author{
Perspective of legal genres and feminisms in \\ the teaching of law \\ Perspectiva de gêneros legais e feminismos \\ no ensino do direito \\ Perspective des genres juridiques et des féminismes \\ dans l'enseignement du droit \\ 法律教学中的性别与法律女性主义透视
}

\author{
Erika Silvina Bauger ${ }^{1}$ | Universidad Nacional de La Plata \\ Revista Derechos en Acción ISSN 2525-1678/ e-ISSN 2525-1686 \\ Año 4/Nº 11 Otoño 2019 (21 marzo a 21 junio), 297-312 \\ DOI: https://doi.org/10.24215/25251678e277 \\ ORCID: https://orcid.org/0000-0002-6207-4203 \\ Recibido: $17 / 03 / 2019$ \\ Aprobado: 14/06/2019
}

Resumen: Este trabajo se origina en una línea de preocupaciones y ocupaciones teóricas y prácticas en nuestra tarea docente en torno a la necesidad de introducir la perspectiva de géneros en la enseñanza del Derecho como conditio sine qua non, para que la igualdad de derechos humanos de las mujeres y otras identidades no hegemónicas, no sea una mera declaración formal sino una realidad tangible y vivenciada en el aquí y ahora. La incorporación de variables de género en el Derecho ha

\footnotetext{
1 Abogada, egresada con Diploma de Honor y Medalla de la UNLP. Premio "Joaquín V. González". Auxiliar Docente con funciones de Adjunta de la Cátedra I de Derecho Internacional Privado de la FCJyS. de la UNLP. Miembro del Instituto de Derecho Internacional Privado del Colegio de Abogados de La Plata. Becaria de investigación en Iniciación, Perfeccionamiento y Formación Superior de SECyT de la UNLP. Maestranda en Relaciones Internacionales y en Derechos Humanos de la UNLP. Estudiante de la Especialización en Docencia Universitaria de la UNLP. Integrante de la Red de Profesoras de la FCJyS y del Observatorio de Enseñanza del Derecho de la UNLP. ebauger@gmail.com ebauger@jursoc.unlp.edu.ar. Orcid: 0000-0002-6207-4203.
} 
puesto en el tapete de manera crítica, las parcialidades descriptivas y prospectivas de intocables teorías y conceptos jurídicos, lo que nos obliga a incorporar un enfoque metodológico de género desde el conocimiento mismo de las bases y fundamentos del Derecho y en especial, de los Derechos Humanos. Esto afecta, de manera indubitable, a nuestra tarea docente, de investigación y de extensión, poniendo en tela de juicio el modelo científico académico que venimos desarrollando, lo que en consecuencia, redundará en la formación de las futuras generaciones de profesionales.

Palabras clave: perspectiva de géneros- feminismos jurídicos- enseñanza- Derecho- Derechos humanos.

Abstract: This work originates in a line of concerns and theoretical and practical occupations in our teaching task around the need to introduce the gender perspective in the teaching of Law as a conditio sine qua non, so that the equality of human rights of women and other non-hegemonic identities, not a mere formal declaration but a tangible reality lived in the here and now. The incorporation of gender variables in the Law has critically addressed the descriptive and prospective biases of untouchable theories and legal concepts, which forces us to incorporate a methodological approach to gender from the very knowledge of the foundations and fundamentals of Law and, in particular, of Human Rights. This affects, undoubtedly, our teaching, research and extension, putting into question the academic scientific model that we have been developing, which consequently will result in the training of future generations of professionals.

Key words: perspective of genders - legal feminisms - teaching - Law Human rights.

Resumo: Este trabalho origina-se em uma linha de preocupações e ocupações teórico-práticas em nossa tarefa de ensinar em torno da necessidade de introduzir a perspectiva de gênero no ensino do Direito como condição sine qua non, para que a igualdade de direitos humanos de mulheres e outras identidades não hegemônicas, não uma mera declaração formal, mas uma realidade tangível vivida no aqui e agora. A incorporação de variáveis de gênero no Direito tem abordado criticamente os vieses descritivos e prospectivos de teorias e conceitos jurídicos intocáveis, o que nos obriga a incorporar uma abordagem metodológica ao gênero a partir do próprio conhecimento das fundações e fundamentos Direito 
e, em particular, dos Direitos Humanos. Isso afeta, sem dúvida, nosso ensino, pesquisa e extensão, colocando em questão o modelo científico acadêmico que temos desenvolvido, o que consequentemente resultará na formação de futuras gerações de profissionais.

Palavras - chave: perspectiva dos gêneros - feminismos legais - ensino - Direito - Direitos humanos.

Résumé: Ce travail a pour origine une ligne de préoccupations et des occupations théoriques et pratiques de notre tâche d'enseignement concernant la nécessité d'introduire la perspective de genre dans I'enseignement du droit en tant que condition sine qua non, afin que l'égalité des droits de I'homme les femmes et d'autres identités non hégémoniques, non pas une simple déclaration formelle mais une réalité tangible vécue ici et maintenant. L'incorporation de variables liées au sexe dans la loi a permis d'attaquer de manière critique les biais descriptifs et prospectifs de théories et de concepts juridiques intouchables, ce qui nous oblige à intégrer une approche méthodologique du genre à partir de la connaissance même des fondements du droit et, en particulier, des droits de I'homme. Cela affecte sans aucun doute notre enseignement, notre recherche et notre vulgarisation, remettant en question le modèle scientifique académique que nous avons développé, qui aboutira par conséquent à la formation de futures générations de professionnels.

Mots clés: perspective des genres - féminismes juridiques - enseignement - Droit - droits de I 'homme.

摘要: 这项工作起源于我们在教学任务中的一系列关注和理论与 实践职业, 围绕在法律教学中引入性别观点的必要性. 将性别变量 纳入法律, 批判性地解决了不可接触的理论和法律概念的描述性和 前瞻性偏见, 迫使我们从基础知识和基础知识中纳入性别方法论方 法。法律, 特别是人权。

关键字: 性别观点 - 法律女权主义 - 教育 - 法律 - 人权。

\section{Marco referencial. Diagnóstico y contexto institucional}

Se busca reflexionar sobre la necesidad de revisar las estrategias, los métodos y los parámetros para reorientar el proceso 
de aprendizaje y enseñanza del Derecho desde una teoría de la argumentación basada en la aplicación concreta de los Derechos Humanos sin discriminación y el de recuperar las teorías del feminismo jurídico en el ámbito en que nos desempeñamos: nuestra Facultad de Ciencias Jurídicas y Sociales de la Universidad Nacional de La Plata.

Los feminismos jurídicos se pueden situar históricamente en la década de los setenta en los Estados Unidos como articulación de los activismos feministas y en sus diversas manifestaciones teóricas: liberales, marxistas, radicales, poscoloniales, postmodernas, entre otras, que generaron distintas estrategias de acción. Asimismo, originaron heterogéneas recepciones en los campos disciplinares como la filosofía, la sociología y la política, conectados también a problemas en torno a casos judiciales y atravesando agendas políticas diferentes según el contexto institucional. Es decir, no podemos hablar de un sólo tipo de feminismo jurídico sino de varios que plantean perspectivas de trabajo situadas en los distintos contextos y ancladas en necesidades específicas de las mujeres y otras identificaciones no hegemónicas LGTBIQ (Lesbianas, Gays, Trans, Bisexuales, Intersexuales, Queer). ${ }^{2}$

\section{Gérmenes de los feminismos jurídicos}

Desde la constitución de los Estados modernos se han producido diversas consideraciones respecto del Derecho y en especial, del objeto y fundamentos de los Derechos Humanos. Los Derechos Humanos y sus antecesores -los Derechos del Hombre y el Ciudadano- surgen en la modernidad. Es inútil

\footnotetext{
2 En los noventa crearon un movimiento apropiándose de la palabra" queer" y convirtiéndola en aquella que identificara un nuevo movimiento en el que el sexo y el género estuvieran en eterna construcción y transformación. Estas personas sintieron que muchos gays y lesbianas querían imitar el modelo de familia heterosexual estadounidense. "El término será cuestionado, remodelado y considerado obsoleto en la medida en que no ceda a las demandas que se oponen a él precisamente a causa de las exclusiones que lo movilizan", sostiene Judith Butler, una de las madres de este movimiento, en su ensayo 'Acerca del término queer'.
} 
rastrear genealogías previas ya que llegan de la mano de la burguesía, del dominio de la naturaleza, de la esperanza en el progreso, del hombre racional y de la dominación y circulación del capital. Es decir, su nacimiento cumplió una función de legitimación de nuevas formas de vida en el Estado moderno y de la configuración de un sujeto en tanto eje del mundo moderno. Sin embargo, al mismo tiempo, abrieron una doble vía revolucionaria, una segunda brecha de revolución que no había sido prevista por los que construyeron el mundo moderno: la que reclama los sueños no cumplidos de todes y cada uno de sus miembros.

Una de las proclamas más importantes del pensamiento ilustrado, es utilizada por las mujeres del siglo de las luces para poner en evidencia las contradicciones de una organización política, social y económica que no beneficia con el principio de igualdad a todos sus integrantes. El status históricamente determinado de sujeto de derecho - macho, mayor, blanco, occidental, heterosexual y propietario- que se esconde dentro del neutro universal de individuo abstracto y autónomo ha sido cuestionado y cambiado, siendo por tanto, modificable. Sin embargo el reconocimiento de derechos a sujetos diversos, y en especial a las mujeres, es todavía dificultoso en la práctica.

Durante el Siglo XX emerge una amplia tradición que comprende al Derecho no sólo en su función social sino también como un fenómeno social. El feminismo jurídico puede ubicarse en esta tradición aunque, previamente a la conformación del campo, los feminismos en sus múltiples expresiones anticiparon dicho enfoque crítico. Se trata de una particular comprensión del Derecho y las estructuras jurídicas modernas, que genera a su vez nuevas claves de lectura de la normativa legal. La comprensión del Derecho como un fenómeno que excede la pura normativa jurídica y se encuentra indisolublemente asociado a lo social, lo cultural y lo político, se advierte en los desarrollos teóricos elaborados por mujeres a partir de la modernidad. El panorama abierto no siguió una única lógica ya que aparecieron múltiples ramas que se combinaron, entremezclaron, 
mantuvieron fieles a sus orígenes o los contrariaron y criticaron radicalmente (Costa Wegsman, 2011).

Desde el momento mismo en que se establecen las formas modernas del Derecho, se comienzan a debatir los elementos centrales del entramado jurídico en su relación con las mujeres, como la igualdad, la ciudadanía y los derechos.

La igualdad de derechos es, entonces, proclamada y reclamada por mujeres en el siglo de la ilustración. Un ejemplo de ello, es Olympe De Gouges, quien junto a otras mujeres lucharon codo a codo con el hombre en la Revolución Francesa de 1789. Redacta la Declaración de los Derechos de la Mujer y la Ciudadana $^{3}$ reproduciendo el texto de la Declaración de los Derechos del Hombre y del Ciudadano sobre el sustrato en él especificado que afirma que "La mujer nace y permanece igual al hombre en sus derechos". De Gouges sostiene que hasta tanto no se produzcan cambios sustanciales en la manera en que las mujeres son concebidas, la promesa de igualdad seguirá su curso sólo en el ámbito de los ideales abstractos. Antes de ser guillotinada por los propios revolucionarios hombres libres franceses, y a pesar pertenecer al "sexo superior tanto en belleza como en coraje", advierte los límites del universal ciudadano y el principio de igualdad.

Otra muestra de ideales feministas, la encontramos paralelamente en Gran Bretaña con Mary Wollstonecraft ${ }^{4}$, quien

3 Declaración de los Derechos de la Mujer y la Ciudadana (redactada en 1789 por Olympe de Gouges para ser decretada por la Asamblea nacional francesa). En el Preámbulo reclama que "Las madres, hijas, hermanas, representantes de la nación, piden que se las constituya en asamblea nacional. Por considerar que la ignorancia, el olvido o el desprecio de los derechos de la mujer son las únicas causas de los males públicos y de la corrupción de 105 gobiernos, han resuelto exponer en una declaración solemne, los derechos naturales, inalienables y sagrados de la mujer...". A partir de aquí reproduce en 17 artículos los derechos consagrados para el hombre en la Declaración de los Derechos del Hombre y el Ciudadano de 1789. Culmina con un Epílogo arengando a las mujeres a despertar y a reclamar sus derechos.

4 Mary Wollstonecraft escribe una de las primeras obras de la literatura y filosofía feministas: "Vindicación de los derechos de la mujer" (1792), cuyo título original en inglés es "A Vindication of the Rights of Woman: with Strictures on Political and Moral Subjects". En 
sostiene que es necesario el abandono del ámbito doméstico por parte de las mujeres para el desarrollo de su lugar en el mundo del trabajo. La equiparación de las posibilidades de hombres y mujeres se postula como una condición sine qua non para los objetivos revolucionarios de una humanidad más libre y racional. Cabe señalar que esta autora reconoce diferencias entre varones y mujeres, sostiene que éstas son resultado del trato y la educación diferencial que unos y otras reciben. Es por ello, que su propuesta se centra en una educación que promueva y potencie la autonomía y la racionalidad de las mujeres, en pos de alcanzar el ideal igualitario. En este entendimiento, Wollstonecraft combate las ideas respecto de una naturaleza inferior de las mujeres en su aspecto racional y moral, y contrapone una noción de igualdad por la cual se vislumbra el modo en que las diferencias son efectos de un orden no natural. Es importante destacar que la base de su argumento centrado en la necesidad de ampliar la educación de las mujeres, marca un hito histórico relevante en el pensamiento feminista: el ideal igualitario se concreta siempre en función de determinadas condiciones materiales que posibilitan o limitan su alcance (Bodelón, 2009).

Estas ideas de las mujeres ilustradas encuentran su eco en el sufragismo de fines del siglo XIX, como una conquista del feminismo basado en la convicción de no existir diferencias sustantivas entre mujeres y hombres. No obstante, las desiguales

\footnotetext{
ella rebate la postura sostenida por los teóricos políticos y educacionales del siglo XVIII de que las mujeres no debían tener acceso a la educación. La autora argumenta que las mujeres deberían recibir una educación acorde a su posición en la sociedad ya que, según la escritora, son esenciales para la nación porque son ellas las que educan a los hijos y porque podrían ser consideradas no sólo meras esposas, sino pares de sus maridos. En lugar de verlas como simples elementos decorativos en la sociedad o bienes con los que comerciar a la hora de acordar un matrimonio, Wollstonecraft mantiene que las mujeres son seres humanos que merecen los mismos derechos fundamentales que los hombres. La autora se decidió a escribir "Los derechos de la mujer" tras leer el informe de 1791 de Charles Maurice de Talleyrand-Périgord para la Asamblea Nacional Constituyente de Francia que sostenía que las mujeres solamente debían recibir educación relacionada con el ámbito doméstico. "Los derechos de la mujer" fue escrito como respuesta directa a los eventos que estaban teniendo lugar. Wollstonecraft tenía la intención de escribir un segundo volumen más meditado, pero murió antes de poder completarlo.
} 
condiciones en el ejercicio efectivo de los derechos, lleva a reconsiderar el concepto jurídico de igualdad de derechos ${ }^{5}$.

Siguiendo a Zuñiga Añazco (2009), el pensamiento feminista nacido como respuesta a las deficiencias de la universalidad moderna, revela hacia mediados del siglo XX fuertes discrepancias internas respecto de los alcances de la igualdad ciudadana. La revisión del concepto afirma que la pretensión de igualdad implica la adaptación de las mujeres a cierto modelo de ciudadano hombre, invisibilizando sus necesidades y deseos para ajustarse a las características del modelo de sujeto de derecho. Esta "segunda oleada" feminista, al decir de Pitch (2010), emerge en los países como Estados Unidos y Europa occidental donde tuvo lugar la emancipación femenina desde el punto de vista jurídico. En los años sesenta ingresan en la escuela y luego en la universidad una gran masa de mujeres, quienes se dan cuenta que la igualdad es sólo formal y lo que se sigue esperando de ellas - a pesar de la educación recibida- es que continúen siendo esposas y madres. La cuestión de la igualdad es, en consecuencia, una cuestión cultural, no sólo jurídica, social y económica.

Este movimiento rechaza una igualdad entendida como asimilación al modelo masculino. Así, los principios de imparcialidad y objetividad en el concepto de igualdad ciudadana son funcionales al modelo patriarcal establecido; el derecho se constituye en una herramienta velada para satisfacer los intereses y necesidades del predominio masculino (Costa Wegsman, 2011). En esta "segunda oleada", las propuestas feministas parten de una premisa fundamental: puesto que el derecho es un producto del patriarcado, es una institución hecha a partir del punto de vista masculino dominante. El derecho es, por tanto, masculino (MacKinnon, 1987) ${ }^{6}$. En la medida en que el

5 El derecho garantiza el acceso al trabajo, sin embargo las mujeres cobran un salario inferior al de los hombres por la misma labor. Asimismo, son las principales responsables del cuidado familiar y del trabajo doméstico, no acceden a puestos jerárquicos y son las principales perjudicadas por acoso laboral.

6 MacKinnon es una jurista académica, abogada, profesora, escritora y activista feminista estadounidense. Especializada en igualdad de género en derecho internacional y 
paradigma de la neutralidad es comprendido como un dispositivo patriarcal, la propuesta para enfrentarla es visibilizar las diferencias entre hombres y mujeres. De tal forma, se empieza a analizar críticamente no sólo cómo el principio de igualdad ha sido entendido por las políticas, sino el principio de igualdad mismo. No se trata sólo de obtener el reconocimiento pleno de los derechos de los que, formalmente ya se es titular, sino de interrogar a la lógica misma de los derechos, a su lenguaje, al sujeto al que son atribuidos. No se trata de la paridad en el mundo dado, sino de reconstruir un mundo que reconozca la existencia de dos sujetos (Pitch, 2010).

\section{Feminismos jurídicos en Latinoamérica}

En América Latina los estudios que vinculan el Derecho con el género y las teorías sobre un feminismo jurídico latinoamericano son más recientes y se asocian con la necesidad de actuar en pos del reconocimiento efectivo de los Derecho Humanos de las mujeres a partir de la década de los noventa. Estos desarrollos teóricos se concretan, en primer lugar, en la ratificación de fuente convencional internacional y en leyes que reglamentan su aplicación a diferentes ámbitos de desarrollo de las mujeres. Así, la CEDAW (Convención para la Eliminación de Todas las formas de Discriminación contra la Mujer) ${ }^{7}$ y la Convención Interamericana para prevenir, sancionar y erradicar la violencia contra la mujer (Convención de Belém do Pará) ${ }^{8}$.

constitucional y en teoría política y legal, fue pionera en reclamar legislación contra el acoso sexual y junto a Andrea Dworkin, creó ordenanzas que reconocían la pornografía como una violación de los derechos civiles. Su trabajo toma componentes del marxismo y se centra en la explotación y el abuso sexual, incluido el acoso sexual, la violación, la prostitución, el tráfico sexual y la pornografía.

7 Convención sobre la eliminación de todas las formas de discriminación contra la mujer. Adoptada y abierta a la firma y ratificación, o adhesión, por la Asamblea General en su resolución 34/180, de 18 de diciembre de 1979. Entrada en vigor: 3 de septiembre de 1981, de conformidad con el artículo 27 (1).

8 La Convención Interamericana para Prevenir, Sancionar y Erradicar la Violencia contra la Mujer conocida también como la Convención Belem do Pará, lugar en que fue adoptada en 
Así, desde la década de los noventa comienza a hablarse en términos de Género y Derecho o de Derechos Humanos y Género, como materias de investigación, y más actualmente y en forma incipiente de feminismos jurídicos.

Cabe advertir, que estas teorías problematizan varios temas desde distintas perspectivas contextualizadas y situadas. Por ello, no aparecen en los programas de estudio de las universidades latinoamericanas. Distinta es la recepción de la categoría de género que sí es reconocida en los centros académicos dando nombre a materias, programas, institutos donde confluyen las investigaciones feministas. ${ }^{9}$

Asimismo, el pensamiento latinoamericano se construye desde su condición periférica respecto de los centros del Norte. Para nuestro caso, el pensamiento feminista latinoamericano se articula en la producción de conocimiento y enfoques originales en un movimiento doble de recepción/resignificación y diferenciación de las ideas de los feminismos estadounidenses y europeos. Esta relación centro-periferia es insoslayable para el conocimiento del Derecho en estos temas, puesto que es en Estados Unidos donde se institucionaliza por primera vez un área de pensamiento jurídico feminista (Costa, Wegsman, 2015).

Hacia mediados de la década de los noventa, la vinculación de la academia estadounidense con profesionales y juristas de América Latina es la llave para la publicación de un libro pionero del pensamiento jurídico feminista latinoamericano. El

\footnotetext{
1994 propone por primera vez el desarrollo de mecanismos de protección y defensa de los derechos de las mujeres como fundamentales para luchar contra el fenómeno de la violencia contra su integridad física, sexual y psicológica, tanto en el ámbito público como en el privado y su reivindicación dentro de la sociedad. Define la violencia contra la mujer, establece el derecho de las mujeres a vivir una vida libre de violencia y la destaca como una violación de los derechos humanos y de las libertades fundamentales.

9 En el Instituto de Cultura Jurídica de la UNLP, se ofrece la Especialización para el Abordaje de las Violencias Interpersonales y de Género, para personas con título de Abogacía, Psicología, Trabajador Social, Lic. en Comunicación, Médico y/o aquellos profesionales que estén en contacto con la problemática sobre el tema de violencia y género de Universidades Nacionales, Privadas o Extranjeras reconocidas.
} 
Programa de Derecho de la Mujer de la Facultad de Derecho de la Universidad de Washington inaugura en 1997 un proyecto que convoca a profesionales del derecho de América Latina para trabajar en torno a la discriminación de género en esta región. Como producto final del proyecto, se publica: Género y derecho, compilación de artículos editada por Alda Facio, jurista costarricense, y Lorena Fries, abogada chilena. En su presentación se señala: "este texto, el primero en su clase, escrito por eruditas del derecho de Latinoamérica" (Grossman y Shalleck, 1999). Allí se compilan artículos de juristas de distintos países, donde se analizan las categorías de género y el concepto de patriarcado, se cuestiona la neutralidad del lenguaje del derecho, la división público/privado, las mujeres y su lugar en las familias y se invoca al feminismo como praxis para pensar y hacer el derecho (Lerussi y Costa, 2018).

En nuestro país son muchos los recorridos teóricos realizados que principian un pensamiento argentino sobre la temática. En la bibliografía se citan algunos trabajos, Ellos constituyen una plataforma de acción para una variedad de trabajos en nuestra Facultad de Ciencias Jurídicas y Sociales de la UNLP en sus tradicionales funciones -docencia, investigación y extensión- pero deconstruidas a la luz de estos desarrollos.

\section{Conclusiones y sugerencias}

Las mujeres tomamos el megáfono y nuestra voz resuena en todas las escalas: global, regional, nacional y local. La palabra feminismo ya no es un término extraño y feminista es quien puede ver la desigualdad y opresión que históricamente vivieron y viven las mujeres y quiere revertir esa situación. La desigualdad se expresa en las diferencias salariales que existen en relación a los varones, en el techo de cristal en las cátedras universitarias, en el trabajo doméstico no remunerado, en el cuidado de niñas y niños y ancianas y ancianos que no son reconocidos como trabajos. La forma más extrema de esa opresión son los feminicidios. En lo que va de este año, hay un 
feminicidio cada 36 horas en Argentina. En total, 38 mujeres han sido asesinadas por motivos de violencia de género en lo que va de 2019, tanto en Buenos Aires como en distintas provincias del país. Esta situación dramática tiene un anclaje cultural que se inserta en una matriz machista que necesitamos deconstruir, desnaturalizar y visibilizar para cambiar la realidad.

La discusión por la legalización del aborto que tuvo lugar en el Congreso de la Nación durante el 2018 y que marcó la agenda de trabajo de muchas organizaciones de mujeres, es una muestra del pensamiento cultural sobre este tema. No se discute el aborto clandestino como problema social, sino se discute el aborto en cuanto tal. Y el aborto se ha transformado en un acta de acusación contra las mujeres transformándolas -en cierto imaginario colectivo- en marimachos egoístas, dueñas de la vida y la muerte y que paradójicamente han producido dos nuevas víctimas: los embriones y los hombres. El razonamiento es perverso: puesto que si las mujeres y los hombres han alcanzado plena igualdad, no se puede excluir a los segundos de decisiones como las referentes al embarazo. Las discusiones parlamentarias traslucen discursos patriarcales que dan cuenta del disciplinamiento del cuerpo femenino ${ }^{10}-\mathrm{y}$ por tanto de su vida-, pudiendo hablarse de una auténtica ausencia de bábeas corpus para las mujeres, mientras no se les reconozca plena responsabilidad en orden a la procreación. Lo que significa que no puede haber igualdad de hombres y mujeres hasta que no se garantice ese reconocimiento.

En mi opinión, y a la luz de los recorridos teóricos y prácticos inaugurados por otras mujeres, sólo sabiendo desde adentro como funciona nuestra Universidad, podemos pensar en formas reales y eficaces para introducir el análisis de género y las teorías del feminismo jurídico en los procesos de aprendizaje y

\footnotetext{
10 Sobre el concepto de disciplinamiento del cuerpo femenino, ver Foucault, Michel (1991). Historia de la sexualidad 1- La voluntad de saber. Buenos Aires: Siglo XXI Editores. Y Foucault, Michel (2018). Historia de la sexualidad 4. Las confesiones de la carne. Buenos Aires: Siglo XXI Editores.
} 
enseñanza de las y los futuros profesionales. La posibilidad de plantear problemas concretos sobre el rol del Derecho, la identificación de normas jurídicas discriminatorias y la aplicación de los dispositivos legales, puede ser una manera de contribuir a repensar las prácticas docentes como prácticas sociales, culturales, políticas e históricamente situadas.

En suma, en el marco de mi participación en la Red de Profesoras de la Facultad de Ciencias Jurídicas y Sociales de la UNLP y el Observatorio de Enseñanza del Derecho, se propone lo siguiente:

- Investigación: recuperando los desarrollos teóricos sintéticamente expuestos, producir teorías sobre el feminismo jurídico argentino y en particular, de nuestra Facultad. Para esto se puede elaborar un repositorio de bibliografía para ofrecer a profesoras y profesores, abierto a todo el público y que sea actualizado periódicamente. El estudio del material bibliográfico permitirá reconstruir genealogías de los feminismo jurídicos, reconocer las investigaciones precedentes y producir nuevos conocimientos. Articular las Ciencias Jurídicas con otras Ciencias Sociales para el desarrollo de estrategias interdisciplinarias creativas.

- Docencia: trabajar en la transversalización de estudios de género y teorías del feminismo jurídico en cada una de las materias que componen el currículo de nuestra Facultad, lo cual promovería que les egresades se conviertan en factores de cambio cultural en la aplicación de un Derecho más justo y el acceso real a la justicia sin discriminación. .Asimismo, propongo una asignatura específica "Género y Derechos Humanos", que podría estar adscripta a la asignatura "Derechos Humanos" del nuevo plan de estudios. La propuesta es que sea interdisciplinar, horizontal entre las y los docentes que participen de las clases y la articulación en forma de Taller. Ello supone favorecer la articulación continua entre teoría y práctica promoviendo la revisión de ambas en su vinculación ${ }^{11}$.

11 En esta línea de trabajo se pueden señalar algunas estrategias que se utilizarán a lo largo del Taller. Las mismas sólo configuran una hipótesis de trabajo:1) Actividades de ubicación 
- Extensión: Se propone la indagación y compilación de trabajos de sistematización de experiencias de extensión universitaria de distintas Casas de Estudio a los fines de revisar críticamente la praxis extensionista y discutir sobre los modelos de extensión universitaria que se visualizan en nuestra Universidad y cómo esto nos conduce en definitiva a debatir sobre el modelo de Universidad que pretendemos para la comunidad. Esto nos permitirá elaborar una propuesta categórica desde lo académico para los procesos de interpelación de una institución en contexto en temáticas relativas al género y a los Derechos Humanos de las mujeres y otras identidades no hegemónicas. La profundización de los procesos de extensión universitaria no son solamente un camino adecuado sino, más bien, es el comienzo del camino que debemos transitar hacia el espacio de integralidad, donde simplemente la docencia, investigación y extensión sean cotidianas en la formación en contexto y donde los procesos de extensión alimenten a las líneas de enseñanza y la investigación, y viceversa. Porque, en este firme desafío, encontraremos la democratización del conocimiento generado en situaciones concretas de vida de la comunidad. Que la falta de Pueblo en nuestra Universidad y en nuestra Facultad no se naturalice, porque estaremos deslegitimizando una institución que amamos y como ciudadanas y ciudadanos contenemos.

Quiero recordar que cuando Lito Nebbia, escribió: "Si la historia la escriben los que ganan eso quiere decir que hay otra historia", no se equivocó. Bajo el aparente manto de la igualdad concebido históricamente como universalidad, se encubren

\footnotetext{
y apertura al tema propuesto. Presentación de los problemas centrales que se pretende trabajar. Ubicación de las perspectivas teóricas que se recuperan para abordarlo, situando los aportes de la bibliografía. 2) Estrategias de problematización de los temas propuestos en función de relevar las visiones y perspectivas de los alumnos. 3) Instancias de trabajo en subgrupos para el abordaje de situaciones problemáticas y análisis de casos prácticos. 4) Instancias plenarias de integración para la puesta en común de aspectos relativos a la producción de los sub-grupos que permitan sintetizar un mapa de categorías y sus relaciones, reflexionando sobre los aportes a la comprensión de situaciones de aprendizaje y delineando posibles perspectivas y criterios de intervención en las mismas.
} 
profundas y terribles desigualdades. Necesitamos recuperar esa "otra" historia: la silenciada, la negada, la dormida, la ausente. Por eso, finalizo citando el epílogo de la Declaración de los Derechos de la Mujer y la Ciudadana redactado por Olympe De Gouge en 1789: "Mujer, despierta; el rebato de la razón se hace oír en todo el universo; reconoce tus derechos. El potente imperio de la naturaleza ha dejado de estar rodeado de prejuicios, fanatismo, superstición y mentiras. La antorcha de la verdad ha disipado todas las nubes de la necedad y la usurpación. El hombre esclavo ha redoblado sus fuerzas y ha necesitado apelar a las tuyas para romper sus cadenas. Pero una vez en libertad, ha sido injusto con su compañera. ¡Oh, mujeres! ¡Mujeres! ¿Cuándo dejaréis de estar ciegas? ¿Qué ventajas habéis obtenido de la revolución? Un desprecio más marcado, un desdén más visible. [...] Cualesquiera sean los obstáculos que os opongan, podéis superarlos; os basta con desearlo."

\section{Bibliografía}

BERGALLO, Paola, JARAMILLO SIERRA, Isabel C. y VAGGIONE, Juan (2018). El aborto en América Latina. Estrategias juridicas para luchar por su legalización y enfrentar resistencias conservadoras. Buenos Aires: Siglo XXI Editores.

BIRGIN, Haydée (2000). El Derecho en el género y el género en el Derecho. Buenos Aires: Biblos.

BIRGIN, Haydée (2006). Acceso a la justicia como garantía de igualdad. Buenos Aires: Biblos.

BODELÓN Encarna (2009). "Feminismo y derecho: mujeres que van más allá de lo jurídico". En: Gemma Nicolás y Encarna Bodelón (comps.), Género y dominación. Críticas feministas del derecho y el poder. Barcelona: Antrhopos.

COSTA WEGSMAN, Malena (2011). Críticas del Derecho y feminismos jurídicos. II Jornadas del Centro Interdisciplinario de Investigaciones en Género, 28, 29 y 30 de septiembre de 2011, La Plata, Argentina. En Memoria Académica. Disponible en:http://www.memoria.fahce.unlp.edu.ar/trab_eventos/ ev.4893/ev.4893.pdf. 
FEMENÍAS, María Luisa (2000). Sobre sujeto y género. Lecturas feministas desde Beauvoir a Butler. Buenos Aires: Catálogos.

FOUCAULT, Michel (1991). Historia de la sexualidad 1- La voluntad de saber. Buenos Aires: Siglo XXI Editores

FOUCAULT, Michel (2018). Historia de la sexualidad 4. Las confesiones de la carne. Buenos Aires: Siglo XXI Editores

GROSSMAN, Claudio y SHALLECK, Ann. "Programa de Derecho de la Mujer y Derecho Internacional". En: FACIO, Alda y FRIES, Lorena. Género y Derecho. Santiago de Chile: LOM, 1999. p. 9 -12 .

LERUSSI, Carla y COSTA, Malena (2018). "Los feminismos jurídicos en Argentina. Notas para pensar un campo emergente a partir de la década de 1990". En: Rev. Estud. Fem. vol.26 no.1 Florianópolis 2018 Epub Jan 15, 2018. Disponible en: http://dx.doi. org/10.1590/1806-9584.2018v26n141972

OLSEN, Frances (1999). "El sexo del derecho". En: RUIZ, Alicia. Identidad femenina y discurso jurídico. Buenos Aires: Biblos.

MACKINNON, Catherine (1987). Feminism Unmodified. Feminismo inmodificado. Discursos sobre la vida y el derecho. Buenos Aires: Siglo XXI Editores, 2014.

PITCH, Tamar (2010). "Sexo y género de y en el derecho: el feminismo jurídico". En: Un panorama de filosofía jurídica y politica: (50 años de "Anales de la Cátedra Francisco Suárez"). Italia: pp. 435-460.

RODIGOU NOCETTI, Maite, BLANES, Paola, BURIJOVICH, Jacinta y DOMINGUEZ, Alejandra (2011). Trabajar en la Universidad. (Des) Igualdades de género por transformar. Córdoba: Universidad Nacional de Córdoba Editorial.

RUIZ, Alicia (2000). Identidad femenina y discurso jurídico. Buenos Aires: Biblos.

SMART, Carol (1992). "La teoría feminista y el discurso jurídico". En: BIRGIN, Haydée. El Derecho en el género y el género en el Derecho. Buenos Aires: Biblos.

ZÚÑIGA AÑAZCO, Yanira (2009). “La 'generización' de la ciudadanía. Apuntes sobre el rol de la diferencia sexual en el pensamiento feminista". En: Revista de Derecho Universidad Austral de Chile, V. XXII/ No 2 (diciembre 2009), ps. 39-64. Disponible en: http://dx.doi.org/10.4067/S0718-09502009000200003 